\title{
Vacinação contra influenza no enfrentamento da COVID-19: integração ensino-serviço para formação em enfermagem e saúde
}

\author{
Vaccination against influenza in the face of COVID-19: teaching-service integration for training in nursing \\ and health \\ Vacunación contra influenza en el enfrentamiento de la COVID-19: integración enseñanza-servicio para
}

Anna Maria Meyer Maciel Rodríguez ${ }^{1}$ (D) Tauani Zampieri Cardoso² (1) Patricia Abrahão-Curvo² (1) Larissa Gerin ${ }^{3}$ (1) Pedro Fredemir Palha ${ }^{2}$ (D) Susana Inés Segura-Muñoz ${ }^{2}$

1. Universidade de São Paulo, Escola de Enfermagem de Ribeirão Preto, Programa de Pós-Graduação em Enfermagem em Saúde Pública. Ribeirão Preto, SP, Brasil.

2. Universidade de São Paulo, Escola de Enfermagem de Ribeirão Preto, Departamento de Enfermagem Materno-infantil e Saúde Pública. Ribeirão Preto, SP, Brasil.

3. Prefeitura Municipal de Ribeirão Preto, Secretaria Municipal de Saúde, Divisão de Vigilância Epidemiológica. Ribeirão Preto, SP, Brasil.

Autor correspondente:

Susana Inés Segura-Muñoz.

E-mail:susis@eerp.usp.br.

Recebido em 09/09/2020.

Aprovado em 15/12/2020.

DOI:https://doi.org/10.1590/2177-9465-EAN-2020-0379

\section{Resumo}

Objetivo: descrever a experiência de integração ensino-serviço durante a primeira etapa da $22^{a}$ Campanha Nacional de Vacinação contra a Influenza na emergência da COVID-19. Método: relato de experiência sobre a parceria de um curso de enfermagem de uma das instituições de ensino superior de Ribeirão Preto-SP, Brasil, com serviços da Atenção Primária à Saúde na referida campanha, no período de março a abril de 2020. Resultados: essa parceria mobilizou diversos voluntários para implementar 35 postos volantes de vacinação, incluindo um drive-thru. Nessa primeira etapa, foram aplicadas 91.697 doses do imunobiológico em idosos, de um total de 98.189 , que correspondeu a $83,3 \%$, valor muito próximo da cobertura vacinal de $90 \%$ esperada para esse grupo populacional. Com a parceria, o número total de doses aplicadas em idosos ao final da $22^{\mathrm{a}}$ campanha, na cidade, superou em $42,6 \%$ a média dos últimos cinco anos. Conclusão e implicações para a prática: atribui-se esse percentual ao trabalho coletivo e multiprofissional e ao compromisso social das instituições envolvidas com a proteção da saúde, a preservação da vida e o fortalecimento do Sistema Único de Saúde.

Palavras-chave:Vacinas contra influenza; Enfermagem; Infecções por coronavírus; Atenção primária à saúde; Instituições de ensino superior

\section{Aвstract}

Objective: to describe the experience of teaching-service integration during the first stage of the $22^{\text {nd }}$ Brazilian National Influenza Vaccination Campaign in the emergence of COVID-19. Method: this is an experience report on the partnership of a nursing course from one of the higher education institutions in Ribeirão Preto-SP, Brazil, with Primary Health Care services in that campaign, from March to April 2020. Results: this partnership mobilized several volunteers to implement 35 vaccination stations, including a drive-thru. In this first stage, 91,697 doses of the immunobiological agent were applied to older adults, from a total of 98,189, which corresponded to $83.3 \%$, a value very close to the $90 \%$ vaccination coverage expected for this population group. With the partnership, the total number of doses applied to older adults at the end of the $22^{\text {nd }}$ campaign in the city exceeded the average of the last five years by $42.6 \%$. Conclusion and implications for practice: this percentage is attributed to the collective and multiprofessional work and the social commitment of the institutions involved with protection of health, preservation of life and strengthening of the Unified Health System.

Keywords: Influenza vaccines; Nursing; Coronavirus infections; Primary health care; Higher education institutions.

\section{Resumen}

Objetivo: describir la experiencia de integración enseñanza-servicio durante la primera etapa de la 22ª Campaña Nacional de Vacunación contra el Influenza en la emergencia de la covid-19. Método: un relato de experiencia de la asociación de un curso de enfermería de una institución de enseñanza superior de Ribeirão Preto-SP, Brasil con servicios de la atención primaria a la salud en la referida campaña, en el período de marzo a abril de 2020. Resultados: esta asociación movilizó a varios voluntarios para implementar 35 estaciones de vacunación, incluyendo un drive-thru. En esa primera etapa, fueron aplicadas 91.697 dosis del inmunobiológico en ancianos, de un total de 98.189, que correspondió a 83,3\%, valor muy próximo de la cobertura vacunal de $90 \%$ esperada para ese grupo poblacional. Con la asociación, el número total de dosis aplicadas en ancianos al final de la 22a campaña en la ciudad superó en $42,6 \%$ el promedio de los últimos cinco años. Conclusión e implicaciones para la práctica: este porcentaje se atribuye al trabajo colectivo e interprofesional y al compromiso social de las instituciones involucradas con la protección de la salud, la preservación de la vida y el fortalecimiento del Sistema Único de Salud.

Palabras clave: Vacunas contra influenza; Enfermería; Infecciones por coronavírus; Atención primaria de salud; Instituiciones de enseñanza superior. 


\section{INTRODUÇÃO}

A integração ensino-serviço para a formação em saúde é uma forma de promover a aprendizagem discente junto à realidade do sistema de saúde e da sociedade na qual se vive. Transpor barreiras físicas de salas de aula e inserir os alunos nos diversos cenários de prática profissional são estratégias para identificar e compreender as complexas, diversas e reais necessidades dos serviços de saúde, famílias, pessoas e comunidades. Nesse sentido, a formação em saúde deve estar comprometida com o processo ensino-aprendizagem, com a construção da identidade profissional, a produção de cuidados contextualizados e a resolutividade dos serviços de saúde. ${ }^{1}$

No âmbito da Atenção Primária à Saúde (APS), a produção acadêmica de conhecimentos em saúde-doença-cuidado leva em conta as determinações sociais que incidem sobre o modo de ser e viver das pessoas em territórios adscritos. Esse aspecto interfere também no desenvolvimento das atribuições do enfermeiro e dos membros da equipe, podendo auxiliar a gestão pública de saúde a responder com mais eficácia a diferentes demandas em cenários sanitários específicos. ${ }^{1}$

No atual contexto de pandemia de COVID-19, essa integração pode ser uma ferramenta para ampliar uma das ações coletivas desenvolvidas pela APS junto à população, a vacinação anual contra a gripe sazonal, uma vez que as manifestações clínicas e complicações dessa gripe são muito semelhantes às provocadas pela COVID-19, e ambas podem requerer cuidados intensivos. ${ }^{2}$ Sendo assim, a infecção pelo vírus causador da influenza pode atrasar o diagnóstico pelo coronavírus e contribuir potencialmente com sua disseminação.

A gripe sazonal é uma infecção respiratória aguda de alta prevalência, transmissibilidade e letalidade, sobretudo em idosos e portadores de doenças crônicas, ${ }^{3}$ e a vacinação anual é a medida mais eficaz para diminuir o número de casos, complicações e internações. ${ }^{4}$ Dentre os diversos tipos de vacinas contra influenza, a utilizada no Brasil em 2020 foi a trivalente combinada, fragmentada e inativada, constituída pelas cepas de um vírus similar ao causador da influenza H1N1, H3N2 e linhagem B/Victoria. ${ }^{5}$

Dada a relevância da gripe sazonal frente à pandemia de COVID-19, o Ministério da Saúde antecipou a realização da $22^{\text {a }}$ Campanha de Vacinação contra a Influenza em todo o território nacional. Essa decisão governamental visou conter a circulação do vírus causador da gripe, concomitantemente ao da COVID-19, como forma de proteger os grupos de risco, reduzir o número internações e, com isso, diminuir o impacto sobre os serviços de saúde. ${ }^{6}$ Essa ação, somada às medidas não farmacológicas, faz parte de um conjunto de intervenções em desenvolvimento global de alcance individual, ambiental e comunitário para mitigar a morbimortalidade por infecções respiratórias sazonais e pandêmicas. ${ }^{7}$

Desde a declaração de emergência em saúde pública, feita pela Organização Mundial da Saúde, e a notificação do primeiro caso de COVID-19 na capital paulista no final do mês de fevereiro de 2020, a Secretaria Municipal de Saúde de Ribeirão Preto
(SMS/RP) instituiu o Comitê Técnico de Contingenciamento COVID-19. Esse comitê, constituído por profissionais de saúde, gestores e técnicos dos Departamentos de Atenção à Saúde das Pessoas e de Vigilância em Saúde, teve como objetivo desenvolver um plano de contingência para o enfrentamento da doença na cidade. ${ }^{8}$

Esse documento estabeleceu a implementação de medidas sanitárias individuais e coletivas para conter o avanço da doença conforme os distintos cenários epidemiológicos, tendo como base os planos de contingência nacional e estadual, sendo atualizado de acordo com os avanços científicos e a evolução da transmissão da doença no país e no mundo. Dentre as medidas, destacam-se aquelas relativas à assistência em saúde na APS, com foco no nível 1 de alerta: orientação e capacitação de equipes; levantamento de recursos humanos e materiais, além da elaboração de protocolos e fluxos de atendimento nas unidades de saúde, ${ }^{8}$ para as quais foi incluído o desenvolvimento antecipado da $22^{a}$ Campanha Nacional de Vacinação contra a Influenza.

Haja vista as implicações sociais e sanitárias emergentes da COVID-19, a SMS/RP, por meio da Vigilância Epidemiológica, solicitou a colaboração da Escola de Enfermagem de Ribeirão Preto da Universidade de São Paulo (EERP/USP), uma das instituições de ensino superior da cidade, para participar do planejamento, organização e execução da primeira etapa da 22a Campanha Nacional de Vacinação contra a Influenza, destinada aos idosos. Esse foi um momento que auxiliou no alcance da cobertura vacinal na primeira etapa da campanha, reforçou a integração ensino-serviço e proporcionou maior responsabilidade social aos estudantes de graduação em enfermagem ao ratificar que o cuidado de enfermagem é uma prática social guiada pelas necessidades de uma determinada sociedade, capaz de intervir ativa e positivamente frente aos problemas que se apresentam. ${ }^{9}$

Assim, o objetivo deste trabalho foi descrever a experiência de integração ensino-serviço durante a primeira etapa da 22a - Campanha Nacional de Vacinação contra a Influenza na emergência da COVID-19.

\section{MÉTODO}

Trata-se de estudo descritivo, do tipo relato de experiência sobre a integração ensino-serviço durante a primeira etapa da 22a Campanha Nacional de Vacinação contra a Influenza no município de Ribeirão Preto (SP), que ocorreu no período de 23 de março a 03 de abril de 2020. O município tem 683.777 habitantes, dos quais 110.088 são pessoas com mais de 60 anos, o que representa $16,1 \%$ de idosos do total da população. ${ }^{10}$ Por se tratar de um relato de experiência, dispensou-se apreciação de comitê de ética em pesquisas.

O planejamento das atividades ocorreu no dia 09 de março e foi desenvolvido pelos coordenadores da Vigilância Epidemiológica da SMS/RP juntamente com os enfermeiros docentes e enfermeiros especialistas de laboratório da EERP/USP. As ações planejadas e executadas, bem como as situações vivenciadas em diferentes espaços e momentos da operacionalização da campanha, foram 
registradas em diários de campo pelos atores acima citados, os quais participaram mais intensamente em todas as fases desse processo.

A primeira etapa foi organizada em duas semanas. A primeira semana (de 23 a 27 de março) envolveu 200 profissionais, distribuídos em 35 equipes nos postos volantes de vacinação. A segunda semana (de 30 de março a 3 de abril) contou com 180 profissionais que atuaram em 27 equipes volantes, incluindo um drive-thru disponibilizado em dois dias dessa semana.

Do total de participantes, a universidade contribuiu com mais de 56 voluntários, dentre os quais: 05 enfermeiros docentes, 01 biólogo docente, 01 enfermeiro pesquisador, 03 enfermeiros especialistas de laboratório, 24 alunos de pós-graduação (nível mestrado e doutorado), 21 alunos de graduação em enfermagem cursando o último ano dos cursos de bacharelado e bacharelado e licenciatura em enfermagem e 01 aluno de graduação em ciências farmacêuticas.

\section{RESULTADOS}

Antes de iniciar a primeira etapa da 22ª Campanha Nacional de Vacinação contra a Influenza na modalidade extramuro, foram tomadas medidas sanitárias relativas à imunização com treinamento online ofertado pelos profissionais da Vigilância Epidemiológica da SMS/RP e da EERP/USP aos participantes sobre: conhecimentos atualizados do imunobiológico (incluindo técnica correta de aplicação e condições adequadas de conservação); desenvolvimento da campanha; organização das equipes volantes; uso de equipamentos de proteção individual fornecidos pela universidade; recomendações padrões sobre a COVID-19; identificação de possíveis sintomáticos respiratórios para os quais os participantes indicariam a busca pela unidade de APS mais próxima do posto volante para avaliação e/ou manutenção do distanciamento social com observação da evolução do quadro clínico. Ressalta-se, todavia, que os meios de comunicação e os voluntários envolvidos contribuíram com a divulgação da 22 $2^{\text {a }}$ Campanha Nacional de Vacinação contra a Influenza de forma a incentivar a vacinação nos idosos.

A estratégia utilizada para operacionalizar a primeira etapa da 22 ${ }^{a}$ Campanha Nacional de Vacinação contra a Influenza foi instalar postos volantes de vacinação nas escolas da rede municipal e estadual da educação próximas às unidades de APS face à suspensão temporária do ensino em decorrência da pandemia. Essa medida, pactuada entre os coordenadores da Vigilância Epidemiológica e o gestor da Secretaria Municipal de Educação, favoreceu a implementação do distanciamento de 1,5 m entre as pessoas. A ampliação do espaço físico em áreas abertas das escolas permitiu a organização de filas de acordo com o distanciamento previsto e evitou a aglomeração nas salas de imunização das unidades de saúde.

Para ampliar o acesso à vacinação de idosos com limitações de mobilidade, foi implementado um posto volante de vacinação em sistema drive-thru em área externa de um estádio de futebol. Esse sistema proporcionou maior segurança e conforto aos idosos, acompanhantes e/ou cuidadores, pois o processo de aplicação do imunobiológico foi realizado sem que esse público precisasse sair do veículo.

Em cada um dos postos volantes de vacinação, foi alocada uma equipe constituída pelos representantes da universidade $e$ técnicos de enfermagem das unidades de APS da rede municipal de saúde, escalados por seus supervisores para essa atividade. Esses se revezaram no acolhimento, triagem, organização do fluxo das pessoas, orientações técnicas à comunidade e na aplicação de $0,5 \mathrm{ml}$ da vacina via intramuscular no deltóide - conforme o fluxograma da Figura 1. Ademais, monitoraram a temperatura da caixa térmica entre $2^{\circ} \mathrm{C}$ e $8^{\circ} \mathrm{C}$; aspiraram doses do imunobiológico; descartaram os resíduos adequadamente e orientaram os idosos e familiares quanto aos possíveis efeitos adversos pós-vacinação e à necessidade de atualizar outras vacinas, nas unidades da APS, conforme calendário nacional de imunização. ${ }^{11}$

A parceria entre a Vigilância Epidemiológica da SMS/RP e a EERP/USP contribuiu para o resultado de 91.697 doses aplicadas do imunobiológico em idosos na cidade, de um total de $98.189,{ }^{12}$ que correspondeu a $83,3 \%$, valor muito próximo da cobertura vacinal de $90 \%$ esperada para esse grupo prioritário de pessoas até o final da campanha. ${ }^{4}$ Ressalta-se, ainda, que o número total de doses aplicadas em idosos ao final da campanha no município de Ribeirão Preto superou em 42,6\% a média dos últimos cinco anos. ${ }^{12}$

\section{DISCUSSÃO}

Desde a implantação do Programa Nacional de Imunização, a equipe de enfermagem tem sido a principal responsável pelo planejamento, execução, monitoramento e avaliação de ações, no âmbito da APS, que objetivam ampliar a cobertura vacinal e diminuir a morbimortalidade por doenças imunopreveníveis em uma população. ${ }^{13,14}$

A efetividade dessas práticas depende de um conjunto de competências, como o conhecimento científico sobre imunologia, sobre os fatores que influenciam a resposta imune (relacionados ao vacinado e à vacina), sobre a composição, a via de administração, as interações, as contraindicações dos imunobiológicos e os efeitos adversos esperados pós-vacinação; além disso, o conhecimento técnico e operacional do funcionamento da rede de frio em nível nacional, estadual, municipal e regional (sala de vacina), que inclui o armazenamento, o transporte, a manipulação das vacinas em condições adequadas, desde o laboratório produtor até o momento de aplicação da dose; ainda, registrar doses aplicadas e perdidas, desenvolver busca por faltosos, ações de bloqueio e campanhas para proteger indivíduos e coletividade contra doenças imunopreveníveis. ${ }^{13,15}$

No contexto da APS, as Unidades de Saúde da Família também desenvolvem ações de promoção, prevenção, proteção, diagnóstico, tratamento, reabilitação, redução de danos, cuidados paliativos e vigilância em saúde que são executadas por equipe multiprofissional para uma população definida. Devido às suas especificidades teórico-práticas, a estratégia ainda permite que os profissionais das equipes adentrem nos territórios adscritos às unidades e evidenciem condicionantes e determinantes de 


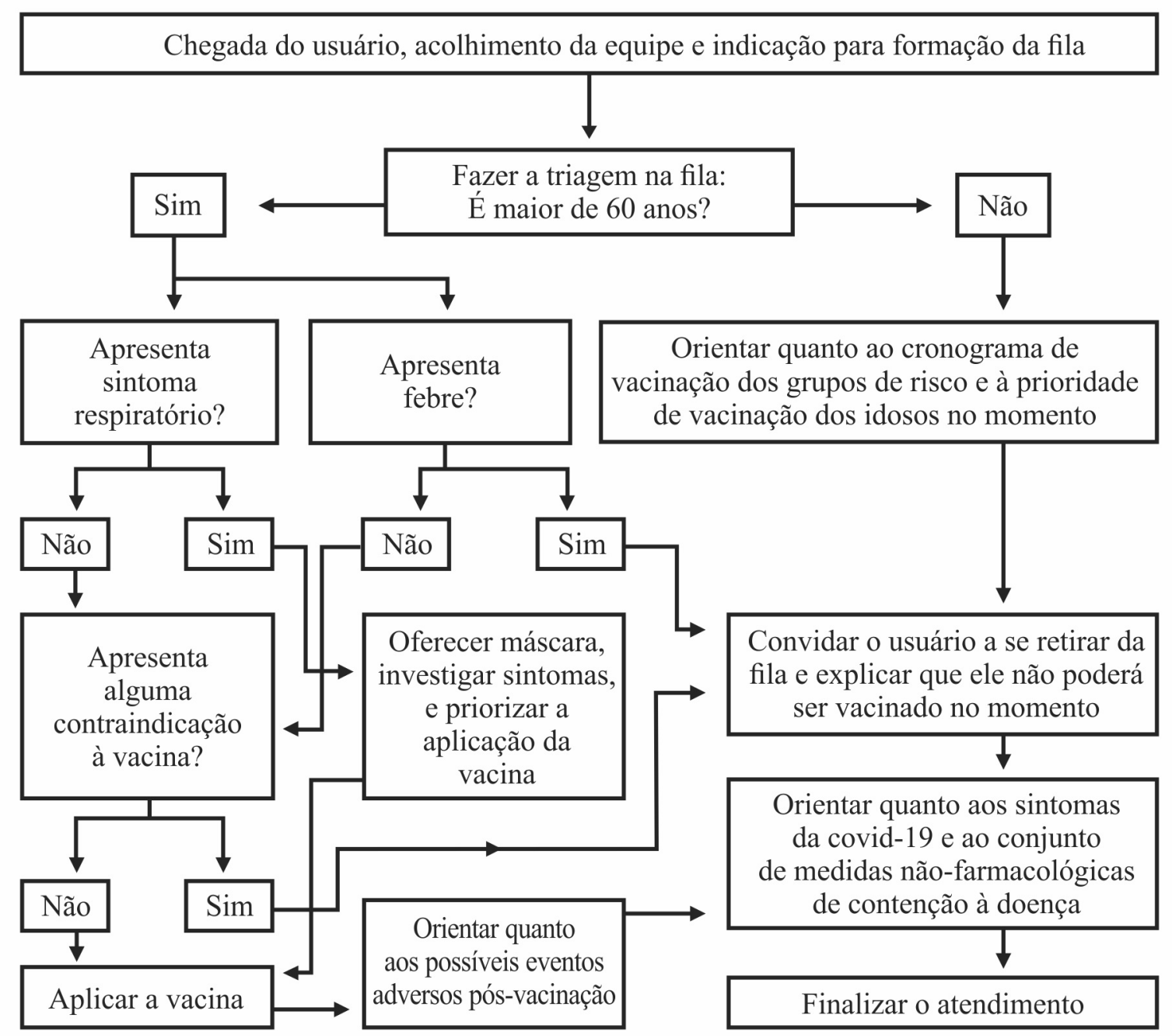

Figura 1. Fluxograma do atendimento na primeira etapa da 22 a Campanha de Vacinação contra a Influenza nos postos volantes no contexto da pandemia da covid-19, Ribeirão Preto, SP, Brasil, 2020.

Fonte: elaborada pelos autores.

saúde. ${ }^{16}$ Essa interação da equipe ao modo de viver da população reforça a necessidade de o conhecimento sobre vacinação ser multiprofissional e não exclusivo dos trabalhadores de enfermagem alocados em sala de vacina. ${ }^{14}$

Para vacinar o maior número possível de idosos durante a primeira etapa da 22 ${ }^{a}$ Campanha Nacional de Vacinação contra a Influenza, os representantes da EERP/USP e coordenadores da Vigilância Epidemiológica da SMS/RP ajustaram condições organizacionais para o trabalho das equipes de vacinação, tomando como base o Planejamento Estratégico em Saúde (PES), que identifica um problema, determina um objetivo, cria um plano factível e administra sua execução. ${ }^{17}$

Nesse sentido, a adoção do PES, em um curto espaço de tempo, permitiu compreender sobre o conceito de explicação situacional e a perspectiva de um olhar abrangente sobre os aspectos de gestão ${ }^{17}$ e de organização para a campanha de vacinação. A utilização do PES, nesse momento, considerou a análise do contexto permeado pela pandemia causada pela
COVID-19, discutiu a viabilidade do plano da campanha e a flexibilidade do mesmo frente a mudanças do cenário sanitário, organização e reorganização das equipes conforme era necessário, focando o planejamento com base nas ações e alcance dos resultados esperados.

Assim, para alcançar a cobertura vacinal esperada em idosos e minimizar os riscos de disseminação da COVID-19, os coordenadores direcionaram as ações conforme o planejado, contornaram imprevistos e promoveram condições adequadas para o trabalho das equipes, respeitando a capacidade assistencial de cada posto volante de vacinação.

Com relação aos postos volantes de vacinação, incluindo o drive-thru, pode-se afirmar que essa estrutura, para além do espaço físico das tradicionais salas de vacina, ampliou o acesso e a acessibilidade de idosos, acompanhantes e cuidadores ao imunobiológico, sugerindo uma certa diminuição de barreiras geográficas que o público com mais de 60 anos frequentemente encontra ao se dirigir às Unidades Básicas de Saúde. ${ }^{18}$ 
A procura pela vacina atingiu percentuais acima do esperado e houve esgotamento das doses, antes do previsto, em diversas cidades brasileiras, incluindo em Ribeirão Preto. Esse fato pode ter relação com a conscientização sobre a importância dessa prática na prevenção de algumas doenças imunopreveníveis ${ }^{19}$ na emergência da COVID-19 e com a implementação de postos volantes operacionalizados por meio do PES. Esse dispositivo, integrante do trabalho gerencial do enfermeiro, ${ }^{17}$ pode ser potencializado pela liderança que esse trabalhador exerce na equipe multiprofissional, sobretudo ao desenvolver habilidades e atitudes relativas à articulação do trabalho assistencial e gerencial nos estabelecimentos de saúde. ${ }^{20}$

Na prática profissional, a liderança é reconhecida no enfermeiro quando esse exercita o diálogo para gerenciar conflitos envolvendo usuários, trabalhadores e gestores quando motiva os trabalhadores a construir um objetivo em comum e quando delega, supervisiona e coordena os cuidados na unidade de saúde e na APS. ${ }^{20}$ Embora a liderança seja entendida como um atributo natural que também pode ser desenvolvido durante o processo de formação em saúde, ${ }^{20}$ não foi o foco deste estudo buscar relações entre essa qualidade e a formação discente durante o período de estágio supervisionado e/ou a integração ensino-serviço no período da campanha.

Atualmente, o exercício da liderança, por parte do enfermeiro, vem sendo associado à oportunização de espaços de colaboração entre os diversos profissionais da equipe que descentraliza funções e atividades, valoriza distintos saberes, estimula a integração dos trabalhadores e o trabalho em equipe, desenhando novas lideranças no trabalho. ${ }^{20}$

No contexto da formação profissional, a articulação da universidade com os serviços de saúde possibilitou ao aluno de graduação e de pós-graduação o desenvolvimento da prática de enfermagem juntamente com outros alunos e profissionais de saúde, materializando o saber no fazer em distintos espaços assistenciais, em uma aproximação do mundo do ensino como mundo do trabalho, ${ }^{1,21}$ sob a ótica colaborativa. Nesse sentido, entende-se que os estabelecimentos de saúde, em especial os da APS, têm forte relação com o processo de formação discente, pois são campos de atuação profissional que oferecem a possibilidade de o aluno aplicar e aprimorar competências construídas em sala de aula.

Destaca-se que a parceria entre a instituição de ensino superior e os serviços de saúde contribuiu para o planejamento, conformação e execução de uma das atividades coletivas de responsabilidade da APS, a campanha de vacinação. Esperase que os conhecimentos teóricos adquiridos na universidade também possam estimular o processo crítico-reflexivo dos alunos sobre as práticas desenvolvidas nos cenários de aprendizagem e estabelecimentos de saúde, visando consolidar os princípios do Sistema Único de Saúde ${ }^{1,22}$ e produzir cuidados de enfermagem e de saúde alinhados aos atributos da APS. ${ }^{23}$

Considerando o resultado positivo obtido com a interação ensino-serviço durante a primeira etapa da $22^{\text {a }}$ Campanha Nacional de Vacinação contra a Influenza, essa ação pode ter contribuído para diminuir os efeitos clínicos e sociais da gripe sazonal nos idosos no contexto da pandemia de COVID-19, uma vez que o envelhecimento contribuiu para o aumento da taxa de mortalidade desse grupo populacional por coronavírus em Wuhan, China. ${ }^{2}$

\section{CONCLUSÃO E IMPLICAÇÕES PARA A PRÁTICA}

Este relato de experiência evidenciou, no contexto da pandemia de COVID-19, a contribuição da integração ensinoserviço no planejamento e na implementação de ações extramuros para atingir $83,3 \%$ de cobertura vacinal em idosos na cidade de Ribeirão Preto durante a primeira etapa da 22a ${ }^{\text {a }}$ Campanha Nacional de Vacinação contra a Influenza. Aponta-se que, ao final da campanha, $89,2 \%$ dos idosos foram vacinados, e esse resultado reflete um trabalho coletivo e multiprofissional, bem como o compromisso social das instituições envolvidas com a proteção da saúde, a preservação da vida e o fortalecimento do Sistema Único de Saúde.

Os resultados indicam, também, contribuições para a área da enfermagem e da saúde, destacando a importância do papel da instituição de ensino superior e dos serviços de APS no processo de formação discente. A limitação desta investigação se refere à impossibilidade de generalizar os achados, pois tanto a parceria estabelecida quanto o número total de doses aplicadas dizem respeito a uma cidade paulista. Ademais, compartilhou-se uma das perspectivas deste trabalho, o que sugere a realização de outros estudos em distintos espaços e contextos para revelar, por exemplo, percepções de usuários do sistema de saúde e de estudantes de enfermagem sobre o impacto da parceria entre instituições de ensino superior e estabelecimentos de saúde do primeiro nível de atenção na prestação de cuidados e na formação profissional, respectivamente.

\section{CONTRIBUIÇÕES DOS AUTORES}

Desenho do relato de experiencia. Anna Maria Meyer Maciel Rodríguez.

Levantamento de fontes e informações. Anna Maria Meyer Maciel Rodríguez. Patricia Abrahão-Curvo.

Análise das informações. Anna Maria Meyer Maciel Rodríguez. Tauani Zampieri Cardoso. Patricia Abrahão-Curvo. Larissa Gerin. Pedro Fredemir Palha. Susana Inés Segura-Muñoz.

Interpretação dos resultados. Anna Maria Meyer Maciel Rodríguez. Tauani Zampieri Cardoso. Patricia Abrahão-Curvo. Larissa Gerin. Pedro Fredemir Palha. Susana Inés Segura-Muñoz

Redação e revisão crítica do manuscrito. Anna Maria Meyer Maciel Rodríguez. Tauani Zampieri Cardoso. Patricia Abrahão-Curvo. Larissa Gerin. Pedro Fredemir Palha. Susana Inés Segura-Muñoz.

Aprovação da versão final do artigo. Anna Maria Meyer Maciel Rodríguez. Tauani Zampieri Cardoso. Patricia Abrahão-Curvo. Larissa Gerin. Pedro Fredemir Palha. Susana Inés Segura-Muñoz 
Responsabilidade por todos os aspectos do conteúdo e a integridade do artigo publicado. Anna Maria Meyer Maciel Rodríguez. Tauani Zampieri Cardoso. Patricia Abrahão-Curvo. Larissa Gerin. Pedro Fredemir Palha. Susana Inés Segura-Muñoz

\section{EDITOR ASSOCIADO}

Gerson Luiz Marinho

\section{REFERÊNCIAS}

1. Brehmer LCF, Ramos FRS. Teaching-service integration: implications and roles in experiences of Undergraduate Courses in Nursing. Rev Esc Enferm USP. 2014;48(1):119-26. http://dx.doi.org/10.1590/S0080623420140000100015 . PMid:24676117.

2. Chen N, Zhou M, Dong X, Qu J, Gong F, Han Y et al. Epidemiological and clinical characteristics of 99 cases of 2019 novel coronavirus pneumonia in Wuhan, China: a descriptive study. Lancet. 2020;395(10223):507-13. http://dx.doi.org/10.1016/S0140-6736(20)30211-7. PMid:32007143.

3. Centers for Disease Control and Prevention. FluView [Internet]. Atlanta: CDC; 2019 [cited 2020 May 12]. Available from: https://www.cdc.gov/ flu/weekly/index.htm

4. El Omeiri N, Azziz-Baumgartner E, Thompson MG, Clará W, Cerpa M, Palekar $R$ et al. Seasonal influenza vaccine effectiveness against laboratory confirmed influenza hospitalizations - Latin America 2013. Vaccine. 2018;36(34):3555-66. http://dx.doi.org/10.1016/j.vaccine.2017.06.036. PMid:28648543.

5. Resolução - RE no 3.076, de 31 de outubro de 2019 (BR). Agência Nacional de Vigilância Sanitária [periódico na internet], 1 nov 2019 [citado 2020 mai 31]. Disponível em: http://portal.anvisa.gov.br/ documents/10181/5684052/RE_3076_2019_.pdf/5647c9cd-153e4302-a637-0066dffe526c

6. Bastos LS, Niquini RP, Lana RM, Villela DAM, Cruz OG, Coelho FC et al. COVID-19 and hospitalizations for SARI in Brazil: a comparison up to the 12th epidemiological week of 2020. Cad Saude Publica. 2020;36(4):e00070120. http://dx.doi.org/10.1590/0102-311x00070120. PMid:32321075.

7. Qualls N, Levitt A, Kanade N, Wright-Jegede N, Dopson S, Biggerstaff $M$ et al. Community mitigation guidelines to prevent pandemic influenza - United States, 2017. MMWR Recomm Rep. 2017;66(1):1-34. http:// dx.doi.org/10.15585/mmwr.rr6601a1. PMid:28426646.

8. Prefeitura Municipal de Ribeirão Preto. Secretaria da Saúde. Plano de contingência para o enfrentamento da covid-19 [Internet]. Ribeirão Preto; 2020 [citado 2020 maio 8]. Disponível em: https://www.ribeiraopreto. sp.gov.br/files/ssaude/pdf/covid-plano-contigencia.pdf

9. Backes DS, Backes MS, Erdmann AL. Promovendo a cidadania por meio do cuidado de enfermagem. Rev Bras Enferm. 2009;62(3):430-4. http://dx.doi.org/10.1590/S0034-71672009000300015. PMid:19597667.

10. Fundação Sistema Estadual de Análise de Dados. Portal de estatísticas do Estado de São Paulo. Perfil dos municípios paulistas [Internet]. São
Paulo: SEADE;2020 [citado 2020 maio 12]. Disponível em: https://perfil. seade.gov.br/

11. Ministério da Saúde (BR). Secretaria de Vigilância em Saúde. Guia de Vigilância em Saúde [Internet]. 3. ed. Brasília: Ministério da Saúde 2019 [citado 2020 maio 31]. Disponível em: http://bvsms.saude.gov. br/bvs/publicacoes/guia_vigilancia_saude_3ed.pdf

12. Ministério da Saúde (BR). Sistema de Informações do Programa Naciona de Imunizações. Vacinômetro [Internet]. Brasília: Ministério da Saúde 2020 [citado 2020 maio 13]. Disponível em: http://sipni-gestao.datasus. gov.br/si-pni-web/faces/relatorio/consolidado/vacinometrolnfluenza.jsf

13. Périco LAD, Wiederkehr PC. Imunizações. In: Ferreira SRS, Périco LAD, Dias VRFG. Atuação do enfermeiro na atenção primária à saúde. Rio de Janeiro: Atheneu; 2017. p. 243-95.

14. Martins JRT, Viegas SMF, Oliveira VC, Rennó HMS. Vaccination in everyday life: experiences indicate Permanent Education. Esc Anna Nery. 2019;23(4):e20180365. http://dx.doi.org/10.1590/2177-9465ean-2018-0365.

15. Ministério da Saúde (BR). Secretaria de Vigilância em Saúde. Manual de rede de frio do Programa Nacional de Imunizações [Internet]. 5. ed. Brasília: $\quad$ Ministério da Saúde; 2017 [citado 2020 out 25]. Disponível em: https://portalarquivos2.saude.gov.br/images/pdf/2017/ dezembro/15/rede_frio_2017_web_VF.pdf

16. Portaria n. 2.436 de 21 de setembro de 2017 (BR). Aprova a Política Nacional de Atenção Básica, estabelecendo a revisão de diretrizes para a organização da Atenção Básica, no âmbito do Sistema Único de Saúde (SUS). Diário Oficial da União, [periódico na internet], Brasília (DF); 2017 [citado 2020 out 25]. Disponível em: http://www.foa.unesp. br/home/pos/ppgops/portaria-n-2436.pdf

17. Ciampone MHT, Tronchim DMR, Melleiro MM. Planejamento e processo decisório como instrumentos do trabalho gerencial. In: Kurcgant $P$, coordenador. Gerenciamento em enfermagem. 3. ed. Rio de Janeiro: Guanabara-Koogan; 2016. p. 33-47.

18. Duarte DC, Oliveira VC, Guimarães EAA, Viegas SMF. Vaccination access in Primary Care from the user's perspective: senses and feelings about healthcare services. Esc Anna Nery. 2019;23(1):e20180250. http://dx.doi.org/10.1590/2177-9465-ean-2018-0250.

19. Hammerschmidt KSA, Santana RF. Health of the older adults in times of the covid-19 Pandemic. Cogitare Enferm. 2020;25:e72849. http:// dx.doi.org/10.5380/ce.v25i0.72849.

20. Lanzoni GMM, Meirelles BHS, Cummings G. Nurse leadership practices in primary health care: a grounded theory. Texto Contexto Enferm. 2016;25(4):e4190015. http://dx.doi.org/10.1590/0104-07072016004190015.

21. Silva MG, Fernandes JD, Teixeira GAS, Silva RMO. Processo de formação $\mathrm{da}(0)$ enfermeira(o) na contemporaneidade: desafios e perspectivas. Texto Contexto Enferm. 2010;19(1):176-84. http://dx.doi.org/10.1590/ S0104-07072010000100021.

22. Kleba ME, Hoefle N, Oliveira GM, Rodrigues OCC. Strengthening the leadership of the Commission on Education-Service Integration for permanent health education. Rev Gaúcha Enferm. 2017;38(4):e20160008. http://dx.doi.org/10.1590/1983-1447.2017.04.2016-0008 PMid:29791540.

23. Starfield B. Atenção Primária: equilíbrio entre necessidades de saúde, serviços e tecnologia. Brasília: Unesco, 2002. 